\title{
Expression of Glutamate Receptor Subtype 3 Is Epigenetically Regulated in Podocytes under Diabetic Conditions
}

\author{
Zhengzhe Li ${ }^{a}$ Haibing Chen ${ }^{b}$ Fang Zhong ${ }^{a}$ Weijia Zhang ${ }^{a}$ Kyung Lee $^{a}$ \\ John Cijiang $\mathrm{He}^{\mathrm{a}, \mathrm{c}}$ \\ ${ }^{a}$ Department of Medicine/Nephrology, Icahn School of Medicine at Mount Sinai, New York, NY, USA; ${ }^{\text {b Department }}$ \\ of Endocrinology and Metabolism, Shanghai Clinical Center for Diabetes, Shanghai Diabetes Institute, Shanghai Key \\ Laboratory of Diabetes Mellitus, Shanghai Jiaotong University Affiliated Sixth People's Hospital, Shanghai, China; \\ 'Renal Section, James J Peters Veterans Affair Medical Center, Bronx, NY, USA
}

\section{Keywords \\ GRIA3 - Glutamate receptor · Diabetic kidney disease · \\ Podocytes · Epigenetics}

\begin{abstract}
Background: Recent studies suggest a role of epigenetics in the pathogenesis of diabetic kidney disease. However, epigenetic changes occurring specifically in kidney cells is poorly understood. Methods: To examine the epigenetic regulation of genes in podocytes under diabetic conditions, we performed DNA methylation and transcriptomic profiling in podocytes exposed to high glucose conditions. Results: Comparative analysis of genes with DNA methylation changes and correspondingly altered mRNA expression identified 337 hypomethylated genes with increased mRNA expression and only 2 hypermethyated genes (ESX1 and GRIA3) with decreased mRNA expression. Glutamate ionotropic receptor AMPA type subunit 3 (GRIA3) belongs to the ionotropic class of glutamate receptors that mediate fast excitatory synaptic transmission in the central nervous system. As podocytes have glutamate-containing vesicles and various glutamate receptors mediate important biological effects in podocytes, we further examined GRIA3 expression and its
\end{abstract}

function in podocytes. Real-time PCR and western blots confirmed the suppression of GRIA3 expression in podocytes under high glucose conditions, which were abolished in the presence of a DNA methyltransferase inhibitor. Sites of DNA hypermethylation were also confirmed by bisulfite sequencing of the GRIA3 promoter region. GRIA3 mRNA and protein expression was suppressed in diabetic kidneys of human and mouse models, and knockdown of GRIA3 exacerbated high glucose-induced apoptosis in cultured podocytes. Conclusion: These results indicate that decreased GRIA3 expression in podocytes in diabetic condition heightens podocyte apoptosis and loss.

(c) 2018 S. Karger AG, Basel

\section{Introduction}

Recent studies suggest that epigenetic regulation, such as DNA methylation, histone modification, and microRNA-mediated gene regulation, play a major role in the pathogenesis of kidney diseases, including diabetic kidney disease (DKD) [1]. Epigenetic alterations in DKD are supported by the Diabetes Control and Complications Trial (DCCT)/Epidemiology of Diabetes Interventions

\section{KARGER}

(c) 2018 S. Karger AG, Basel

E-Mail karger@karger.com

www.karger.com/kdd
John Cijiang $\mathrm{He}, \mathrm{MD} / \mathrm{PhD}$

Division of Nephrology, Box 1243

Icahn School of Medicine at Mount Sinai

One Gustave L. Levy Place, New York, NY 10029 (USA)

E-Mail cijiang.he@ mssm.edu 
and Complications (EDIC) study, in which participants were followed for more than 30 years after being previously assigned to conventional therapy or intensive therapy for diabetes [2]. Participants assigned to conventional therapy continued to develop diabetic complications at significantly higher rates than the intensive therapy group, despite nearly similar $\mathrm{HbA}_{1 \mathrm{c}}$ levels. This persistence of benefit from early therapy suggested "metabolic memory" as a consequence of epigenetic modifications induced by transitory hyperglycemia. A more recent study further demonstrated that DNA methylation differences during the DCCT stage persisted at certain loci associated with hyperglycemia for several years during the EDIC study [3], further supporting the involvement of epigenetic regulation in metabolic memory. However, the mechanisms of epigenetic regulation in DKD are not well understood.

Histologically, the early stage of DKD is characterized by glomerular hypertrophy, glomerular basement membrane thickening, mesangial expansion, glomerular endothelial cell injury, and podocyte loss, while the later stage is characterized by diffuse or nodular glomerulosclerosis, tubulo-interstitial inflammation and fibrosis. Of all of the glomerular morphological characteristics of early diabetic kidneys, the reduction in podocyte density appears to be the strongest predictor of progressive DKD $[4,5]$. Moreover, the degree of podocyte reduction correlates directly with the magnitude of proteinuria $[6,7]$. Therefore, potential therapies in reducing podocyte injury and loss in early diabetic kidneys may be a key approach in reducing albuminuria and in mitigating the DKD progression. However, while several cellular processes have been proposed as potential means of podocyte loss in kidney disease, such as apoptosis, necroptosis, detachment from glomerular basement membrane, and epithelial-mesenchymal transition [8-12], the exact mechanism of podocyte loss in DKD remains unclear.

To determine the epigenetic changes associated with podocyte injury in early diabetic kidneys, we performed genome-wide DNA methylation profiling arrays in conjunction with mRNA sequencing of immortalized human podocytes cultured under normal or high glucose conditions. Glutamate ionotropic receptor AMPA type subunit 3 (GRIA3) was identified as one of the two unique genes that were hypermethylated at the DNA level and correlated with decreased mRNA expression in high glucose condition [13]. Glutamate receptors are heteromeric protein complexes composed of multiple subunits, arranged to form ligand-gated ion channels, which act as predominant excitatory neurotransmitter receptors in the mam- malian brain [14]. As the classification of glutamate receptors is based on their activation by different pharmacologic agonists, the glutamate receptor subtype 3 (GluR3) encoded by GRIA3 belongs to a family of alpha-amino3-hydroxy-5-methyl-4-isoxazole propionate (AMPA)sensitive glutamate receptors. Binding of glutamate to GluR3 induces a conformation change, leading to the opening of the cation channel, and thereby converting the chemical signal to an electrical impulse $[15,16]$. Since recent studies suggest that glutamate receptors also play an important role in podocyte biology [17-21], we posited that the dysregulation of GRIA3 might also lead to podocyte injury and glomerular disease. Therefore, we determined the epigenetic regulation of GRIA3 and its potential relevance in podocyte injury in DKD.

\section{Methods}

\section{Cell Culture}

Immortalized human podocytes were obtained from Dr. Moin Saleem and cultured at $37^{\circ} \mathrm{C}$ for 7 days for full differentiation before being used as described [22].

\section{RNA-Sequencing and DNA Methylation Profiling}

Total RNA was isolated from immortalized human podocytes using TRIzol reagent (Ambion, Life Technologies). Paired-end sequencing with $100 \mathrm{bp}$ read length was carried out on HiSeq 2000 (Illumina Inc.). The reads with good quality were firstly aligned to several human reference databases including the hg19 human genome, exon, splicing junction segment and contamination database including ribosome and mitochondria sequences using the BWA alignment algorithm. After filtering reads mapped to contamination database, the reads that are uniquely aligned to the exon and splicing junction segments with a maximum of 2 mismatches for each transcript were then counted as expression level for the corresponding transcript. The read counts were log2 transformed and normalized at an equal global median value in order to compare the transcription level across samples. The differential analysis by paired LIMMA was performed to identify significantly dysregulated genes in podocytes cultured between high glucose $(\mathrm{HG})$ and normal glucose (NG) at an adjusted FDR $p<0.05$. Total DNA from podocytes was prepared by using spin column (Qiagen) and analyzed on an Illumina $450 \mathrm{~K}$ methylation array by the Laboratory for Translational Genomics at Baylor College of Medicine. The methylation data were processed with open source $\mathrm{R}$ package IMA and MEDME: after data filtering, only the sites on autosomal chromosomes with a significant detection $p$ value $<0.001$ were kept for downstream analysis and then beta was converted to $\log 2$ ratio of beta value/( 1 - beta) value and further smoothened along the genome coordinates to reduce data variation. The differentially methylated sites between HG- and NG-treated podocytes were identified by the paired LIMMA test at $p<0.05$. The gene expression data were correlated with methylation data to identify the differentially expressed genes with the corresponding differential methylation. 


\section{Western Blot}

Cells were lysed in M-PER mammalian protein extraction reagent (ThermoFisher, NY, USA) containing protease inhibitor and tyrosine and serine-threonine phosphorylation inhibitors. Protein was separated on SDA-PAGE and transferred to nitrocellulose membrane. Proteins were detected using specific antibodies: rabbit polyclonal anti-GRIA3 antibody (Life Span Biosciences LSC169522); rabbit polyclonal anti-WT-1 antibody (Santa Cruz C-19); and monoclonal anti- $\beta$-actin antibody (Sigma A5136).

\section{Glomerular RNA Isolation from Mouse Kidneys}

Animal studies were performed in accordance with the guidelines of and approved by the Institutional Animal Care and Use Committee at the Icahn School of Medicine at Mount Sinai (New York, NY, USA). Mice glomeruli were isolated as previously reported [23]. Total RNA was extracted from glomeruli using the Trizol method. Purified RNA underwent DNA digestion using the RNase-free DNase set (Qiagen, 79254). Proteins were extracted using the lysis buffer below for western blot analysis.

\section{Real-Time PCR}

RNA transcript quantification was performed by the 7500 RealTime PCR System. Gene level was normalized to glyceraldehyde 3-phosphate dehydrogenase $(G A P D H)$ and expressed as fold change. The primer sets used for GRIA3 are: $5^{\prime}$-GTGCAGTTTGTCATCCAGATG- $3^{\prime}$ and $5^{\prime}$-ACAGACCTTGCTGTTACTTGC- $3^{\prime}$.

\section{Bisulfite PCR Sequencing}

For bisulfate-treated PCR analysis, $0.5 \mu \mathrm{g}$ genomic DNA was treated with bisulfate by using the EZ DNA methylation-Lightning $^{\mathrm{TM}}$ kit (ZYMORESEARCH) according to the manufactures. PCR was performed using the following primer pairs to amplify the promoter region of GRIA3: forward, $5^{\prime}$-GTTGTAGTTTGAAAGGTTGAT-3' , and reverse, $5^{\prime}$-TTATCAAAACAATCTAAAACTC- $3^{\prime}$. Part of this PCR product was used as template for the nested PCR with the following primer pairs: $5^{\prime}$-AAGGTTGATTTATGTTAGGAG- $3^{\prime}$ and $5^{\prime}$-AAACAATCTAAAACTCTCTCC- $3^{\prime}$. The PCR products (approximately $250 \mathrm{bp}$ in length) were purified and sequenced.

\section{Determination of Apoptosis in GRIA3 Knockdown Human \\ Podocytes}

This was performed using an Expression Arrest GIPZ Lentiviral shRNAmir system (Thermo Scientific, Huntsville, AL, USA). Lentiviral particles were produced by transfecting 293T cells with a combination of lentiviral expression plasmid, $\mathrm{pCD} / \mathrm{NL}-\mathrm{BH} \Delta \Delta \Delta$ packaging plasmid, and VSV-G-encoding pLTR-G plasmid. For viral transduction of the human podocyte cell line, viral supernatants were supplemented with $8 \mu \mathrm{g} / \mathrm{mL}$ polybrene and incubated with cells for a 24 -h period. Cells expressing short-hairpin RNA (shRNA) were selected with puromycin for 2-3 weeks prior to use in all studies. Western blot analysis was performed to confirm the efficiency of knockdown. The apoptosis rate was determined by measurement of caspase- 3 activity by ELISA using a Human Active Caspase-3 Immunoassay kit from R\&D Systems, Inc.

\section{Immunofluorescence}

Kidney sections from mice were prepared accordingly. Immunostaining was performed using a rabbit polyclonal anti-GRIA3 antibody (Life Span Biosciences, LS-C169522). After washing, sec- tions were incubated with a fluorophore-linked secondary antibody (Alexa Fluor 488 anti-rabbit IgG). After staining, slides were mounted in Aqua Poly/Mount (Polysciences Inc.) and photographed under an AxioVision IIe microscope with a digital camera.

\section{Immunohistochemistry}

Archival human biopsy specimens of healthy donor nephrectomies and diabetic nephropathy were collected at the Icahn School of Medicine at Mount Sinai under a protocol approved by the Institutional Review Board. Specimens were initially baked for $20 \mathrm{~min}$ in a 55 - to $60^{\circ} \mathrm{C}$ oven and then processed as described below. Briefly, formalin-fixed and paraffin-embedded sections were deparaffinized, and endogenous peroxidase was inactivated with $\mathrm{H}_{2} \mathrm{O}_{2}$. Sections were then blocked in $2 \%$ goat serum in phosphatebuffered saline for $1 \mathrm{~h}$ at room temperature and then incubated with a rabbit polyclonal anti-GRIA3 antibody (Life Span Biosciences, LS-C169522) at $4{ }^{\circ} \mathrm{C}$ overnight. The next day, sections were washed three times with phosphate-buffered saline and then incubated with secondary antibody for $30 \mathrm{~min}$. Positive staining was revealed by peroxidase-labeled streptavidin and diaminobenzidine substrate with a fixed exposure time of $3 \mathrm{~min}$ for all experiments among the groups. The control included a section stained with only secondary antibody.

\section{Statistical Analysis}

Data are expressed as mean \pm SEM. Unpaired $t$ test was used to analyze data between two groups. The analysis of variance with the Bonferroni post hoc test was used when more than two groups were present. All experiments were repeated at least three times, and representative experiments are shown. Statistical significance was achieved when $p<0.05$.

\section{Results}

\section{High Glucose Alters DNA Methylation and Gene Expression Patterns in Immortalized Human Podocytes}

To characterize high glucose-induced epigenetic and transcriptional dysregulation in podocytes, immortalized human podocytes were exposed to HG (30 mM glucose) or NG control ( $5 \mathrm{mM}$ glucose and $25 \mathrm{~mm}$ mannitol) for 5 days. Methylation profiling was performed using the Infinium HumanMethylation450K BeadChip, and to correlate the DNA methylation changes with mRNA expression levels, RNA sequencing was performed in parallel. Correlation of DNA methylation changes and mRNA expression in HG-treated podocytes identified 337 genes that were hypomethylated and upregulated (Fig. 1). Interestingly, only two genes, ESX1 and GRIA3, were hypermethylated and correspondingly downregulated. Pathway analysis of the total of 339 genes showed that altered genes are largely involved in cell division and cell cycle regulation (Table 1). For two hypermethylated genes in our analysis, ESX1, a homeodomain protein, was report- 
Table 1. Gene ontology (GO) enrichment analysis of 337 genes with corresponding DNA methylation and mRNA expression changes

\begin{tabular}{lrrr}
\hline GO category & GO ID & Genes, $n$ & $p$ value \\
\hline Cell division & GO:0051301 & 20 & $3.74 \mathrm{E}-07$ \\
RNA processing & GO:0006396 & 25 & $1.05 \mathrm{E}-05$ \\
DNA metabolic process & GO:0006259 & 22 & $8.50 \mathrm{E}-05$ \\
ncRNA metabolic process & GO:0034660 & 12 & $1.42 \mathrm{E}-03$ \\
Mitotic spindle organization & GO:0007052 & 4 & $1.69 \mathrm{E}-03$ \\
Chromosome segregation & GO:0007059 & 7 & $2.08 \mathrm{E}-03$ \\
Establishment of protein localization & GO:0045184 & 24 & $3.71 \mathrm{E}-03$ \\
Golgi vesicle transport & GO:0048193 & 8 & $5.71 \mathrm{E}-03$ \\
Regulation of cytoskeleton organization & GO:0051493 & 8 & $6.97 \mathrm{E}-03$ \\
Transcription, DNA-dependent & GO:0006351 & 12 & $8.62 \mathrm{E}-03$ \\
Positive regulation of cell cycle & GO:0045787 & 5 & $1.39 \mathrm{E}-02$ \\
Cellular macromolecule catabolic process & GO:0044265 & 21 & $1.53 \mathrm{E}-02$ \\
Ubiquitin-dependent protein catabolic process & GO:0006511 & 10 & $1.81 \mathrm{E}-02$ \\
Protein complex biogenesis & GO:0070271 & 15 & $3.76 \mathrm{E}-02$ \\
Establishment or maintenance of cell polarity & GO:0007163 & 4 & $4.58 \mathrm{E}-02$ \\
Protein modification by small protein conjugation or removal & GO:0070647 & 7 & $4.75 \mathrm{E}-02$ \\
Apoptosis & GO:0006915 & 16 & $4.89 \mathrm{E}-02$ \\
\hline
\end{tabular}

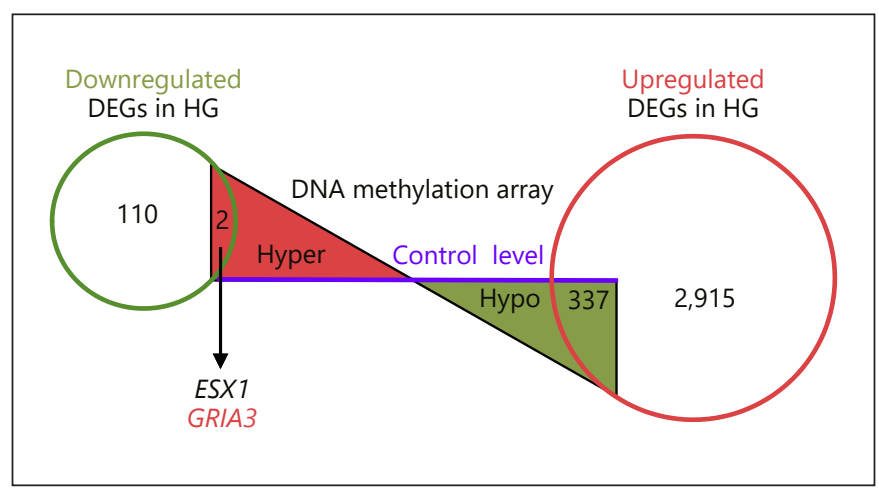

Fig. 1. Comparison of DNA methylation and mRNA expression profiles in human podocytes cultured in high or normal glucose conditions. DNA methylation arrays and RNA sequencing were performed in immortalized human podocytes cultured under normal glucose (NG, $5 \mathrm{~mm}$ glucose and $25 \mathrm{~mm}$ mannitol) or high glucose (HG, $30 \mathrm{~mm}$ glucose) for 5 days. Comparative analysis of changes in mRNA expression with DNA methylation in HG versus NG is shown. There were 337 hypomethylated genes with upregulated mRNA expression and only 2 hypermethylated genes (ESX1 and GRIA3) with downregulated mRNA expression. DEGs, differentially expressed genes.

ed to regulate cell cycle progression and transcription [24-26], and GRIA3 has been shown to promote proliferation in nonneuronal cells $[27,28]$. As other glutamate receptors, such as NMDA receptor, have an important function in podocyte biology and glomerular diseases, we selected GRIA3 for further analysis.

\section{GRIA3 Is Epigenetically Regulated by HG High}

Glucose in Podocytes

We first confirmed that HG conditions suppressed GRIA3 expression in cultured podocytes by real-time PCR and western blot analysis. As shown in Figure 2a-c, HG condition reduced GRIA3 mRNA and GluR3 protein levels. However, pretreatment with DNA methyltransferase inhibitor 5-Aza-2'-deoxycytidine (Aza) restored its expression HG-treated podocytes (Fig. 3a, b). Interestingly, increased GRIA3 expression was associated with an increased expression of podocyte marker Wilms tumor 1 (WT1) (Fig. 3a-c). Bisulfate sequencing revealed four additional DNA methylation sites on GRIA3 promoter in response to HG treatment in cultured podocytes (Fig. 3d). Together, these results indicate the suppression of GRIA3 via DNA methylation in diabetic conditions. Whether the increase in WT1 expression is also controlled epigenetically by DNA methylation or regulated through GluR3 in podocytes remains to be determined.

\section{Reduced GRIA3 Expression in Animal Models and Humans with DKD}

We next examined the expression of GluR3 in kidneys of diabetic mice. Real-time PCR and western blot analysis of isolated glomeruli from streptozotocin (STZ)- 

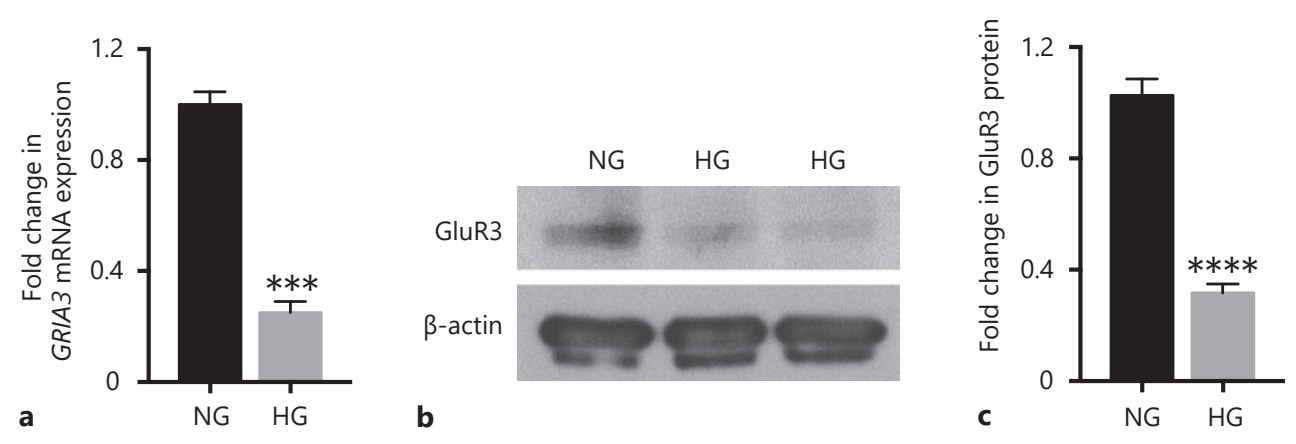

Fig. 2. High glucose (HG) condition suppresses GRIA3 expression cultured human podocytes. a Real-time PCR analysis of GRIA3 in cultured podocytes under HG condition. GRIA3 expression in HG condition is shown as a fold change relative to normal glucose (NG) condition. b Western blot analysis of GluR3 in podocytes treated with NG or HG. $\beta$-Actin was used as a loading control. Densitometric analysis of western blots are shown in c. All experiments were repeated for at least three times; ${ }^{* * *} p<0.001$ and ***** $p<0.0001$ as compared to NG, $n=3$.

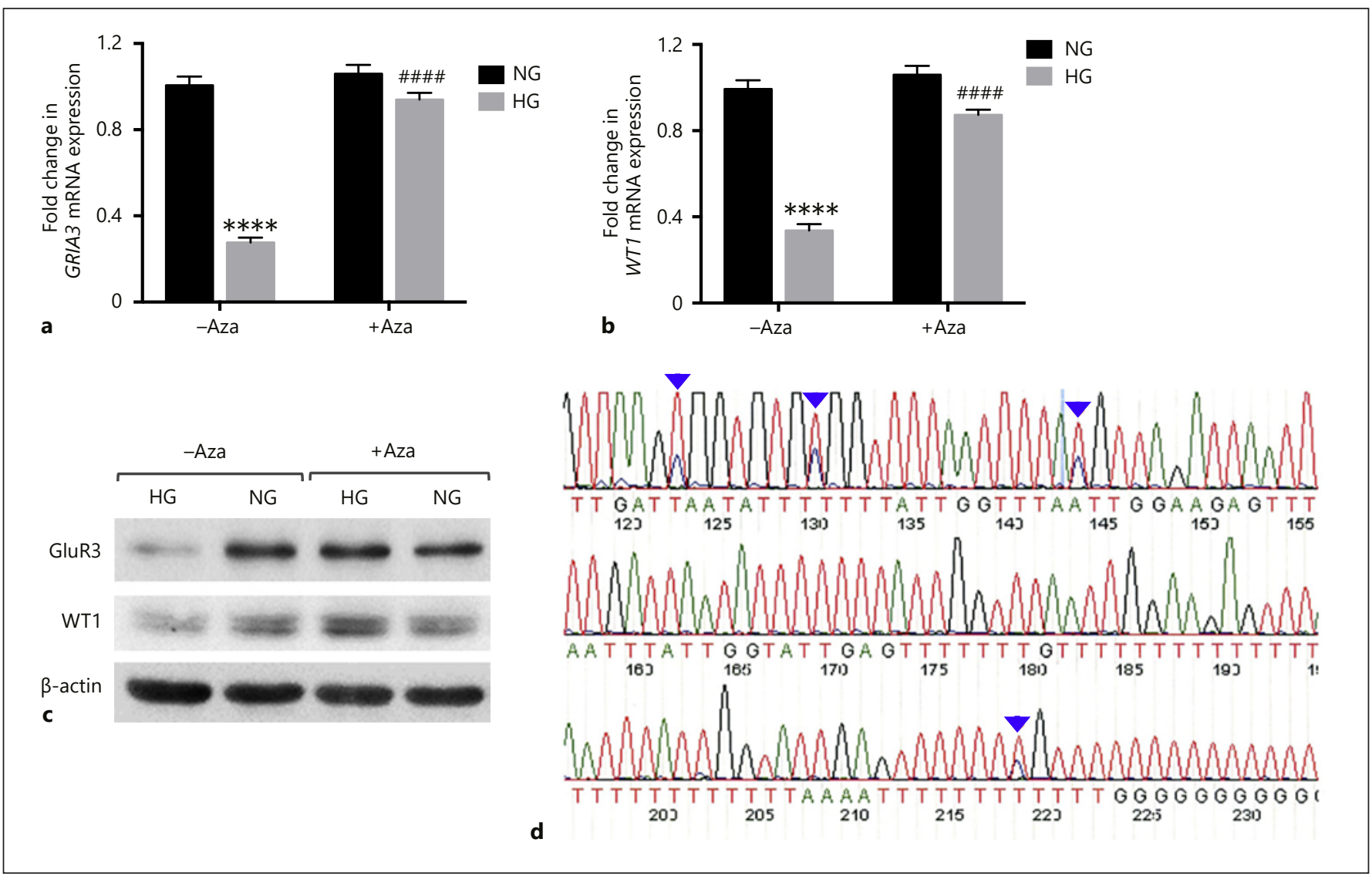

Fig. 3. Inhibition of DNA methylation restores GRIA3 expression in high glucose (HG)-treated podocytes. Immortalized human podocytes were cultured with either normal glucose (NG) or HG as above, with or without DNA methyltransferase inhibitor 5-Aza-2'-deoxycytidine (Aza;1 mM). a Real-time PCR analysis of GRIA3. b Real-time PCR analysis of WT1. c Western blot analysis of GluR3 and WT1. $\beta$-Actin was used as a loading control. d Se- quencing of the GRIA3 promoter region following the bisulfite digestion and PCR amplification shows new DNA methylation sites (indicated by arrowheads) in HG-treated podocytes. All experiments were repeated at least three times. ${ }^{* * * *} p<0.0001$ as compared to NG and ${ }^{\# \# \#} p<0.0001$ as compared to HG-treated without Aza, $n=3$. 
Fig. 4. GRIA3 expression was suppressed in the glomeruli of diabetic mice. Kidney sections and isolated glomeruli were obtained from control (control-eNOS ${ }^{-/}$) and diabetic (STZ-eNOS ${ }^{-/}$) mice as described previously [27]. a Real-time PCR of Gria3 in isolated glomeruli is shown as a relative fold change to control mice. Experiments were repeated at least 3 times. ${ }^{* * * *} p<$ 0.0001 as compared to control mice. $n=5$. b Western blot of GluR3 in isolated glomeruli from 2 mice in each group. c Representative images of GluR3 immunostaining is shown ( $\times 400$ magnification). Glomeruli are outlined with white dotted circle. d Gria 3 mRNA expression in diabetic db/ $\mathrm{db}$ mice in comparison to $\mathrm{db} / \mathrm{m}$ (nephroseq dataset; nephroseq.org), $p=0.005, n=$ 10.

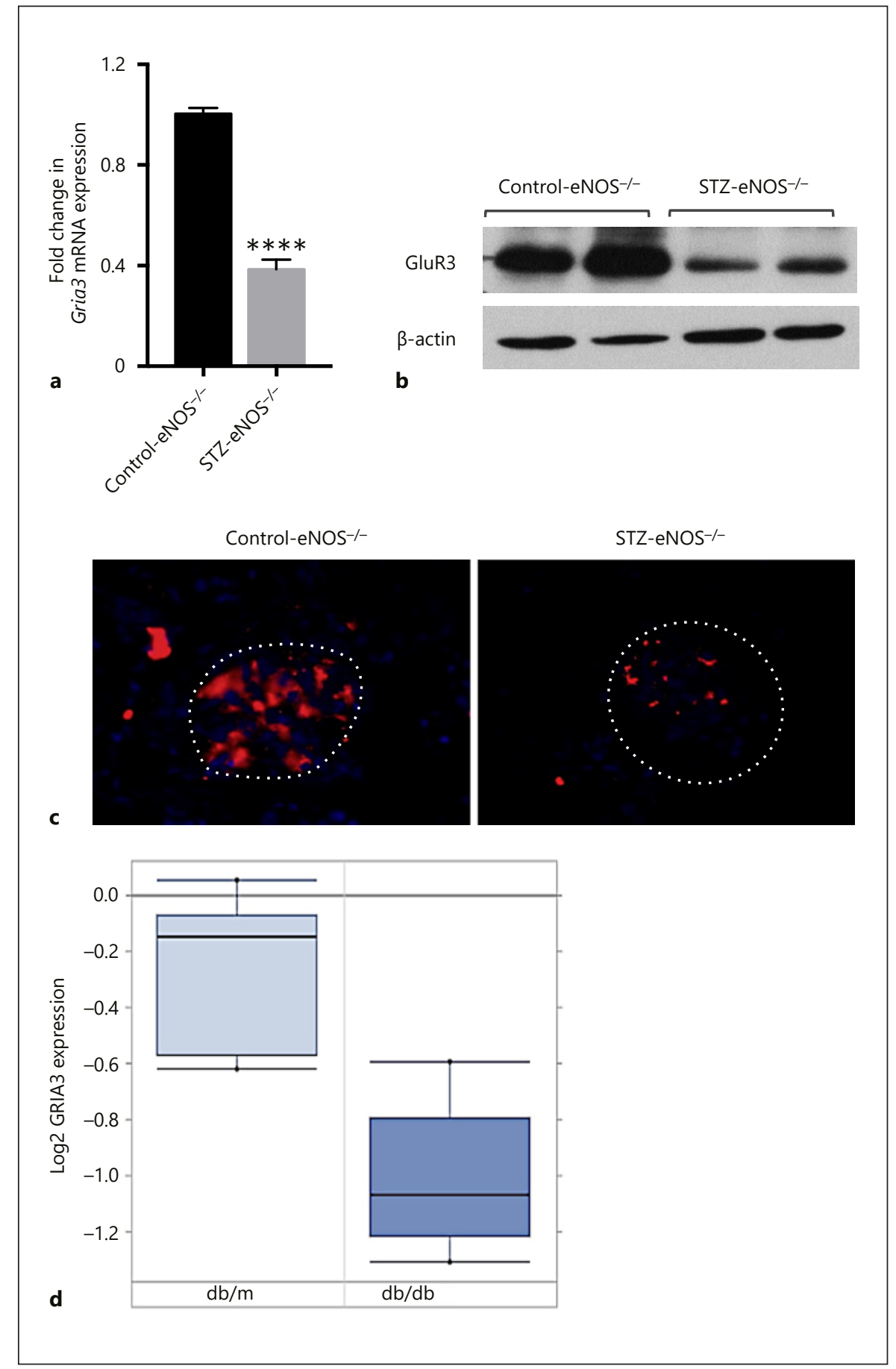

induced diabetic eNOS-deficient (STZ-eNOS ${ }^{-/}$) mice [23] showed significantly reduced GluR3 expression in diabetic mice compared to nondiabetic control eNOS ${ }^{-/-}$ mice (Fig. 4a, b). Immunofluorescence staining on paraffin-embedded kidney sections similarly showed reduced GluR3 expression in the glomeruli of diabetic eNOS ${ }^{-/-}$ mice (Fig. 4c). Consistent with these results, decreased Gria3 mRNA levels were noted in diabetic $d b / d b$ mice as compared to control $\mathrm{db} / \mathrm{m}$ mice according to the Nephroseq database (nephroseq.org) (Fig. 4d). Importantly, immunostaining showed reduced GluR3 expression in human diabetic kidney as compared to nephrectomy sam- 


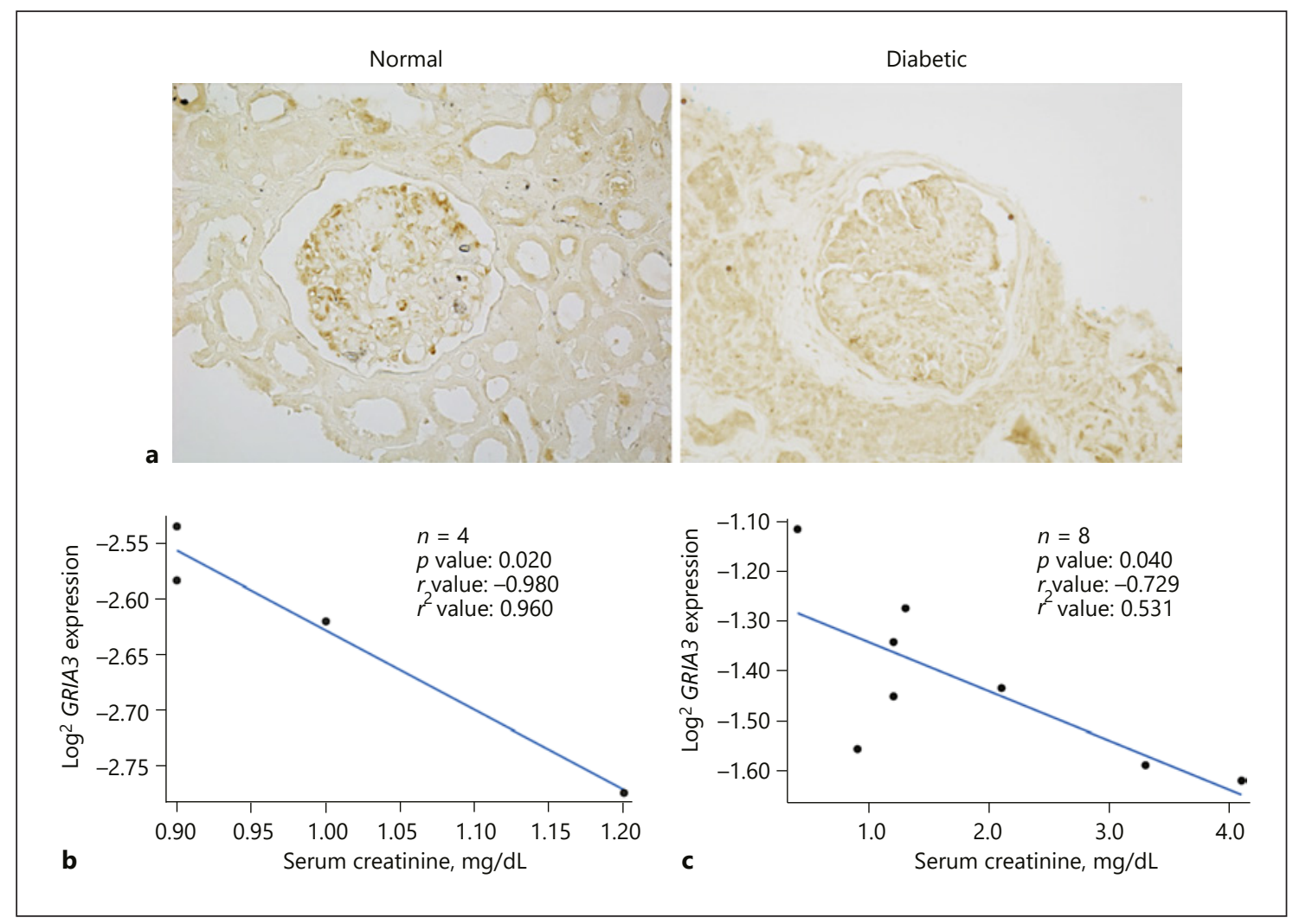

Fig. 5. GRIA3 expression is reduced in human diabetic kidneys. a Representative immunostained images for GluR3 in human kidney biopsies from patients with DKD (diabetic) and nephrectomy (normal) samples. b, c Correlation analysis between GRIA3 mRNA and serum creatinine in glomeruli of CKD patients (b; Ju CKD glom, nephroseq.org) and in focal segmental glomerulosclerosis (FSGS) patients (c; Hodgin FSGS glom, nephroseq.org).

\section{Discussion}

GRIA3 mRNA expression was negatively correlated with serum creatinine in patients with chronic kidney disease (CKD) and with focal segmental glomerulosclerosis (nephroseq.org; Fig. 5b, c), suggesting a potential role of GRIA3 in the progression of kidney disease.

\section{GRIA3 Knockdown Induces Apoptosis in Cultured Human Podocytes}

We then examined the biological effects of reduced GRIA3 expression in cultured human podocytes. Podocytes were stably transduced with lentivector expressing shRNA against GRIA3 or scrambled shRNA control. Figure 6a shows the efficiency of GRIA3 knockdown (Fig. 6a). We then observed its effect in podocytes under HG conditions. GRIA3 knockdown induced podocyte apoptosis under NG condition and exacerbated it under HG condition, as measured by active caspase- 3 activity (Fig. 6b). These results suggest that GRIA3 protects against podocyte apoptosis in DKD.
Although substantial progress has been made in the field of epigenetic regulation in kidney disease, it largely remains limited to datasets obtained from peripheral blood samples. The largest scale epigenome-wide association study thus far examined nearly half a million unique methylation sites in peripheral blood samples of $255 \mathrm{CKD}$ patients and 152 control individuals [29]. Twenty-three tween cases and controls, of which 6 candidate genes were known to be involved in kidney disease, namely: CUX1, ELMO1, FKBP5, INHBA-AS1, PTPRN2, and PRKAG2. Notably, of these 6 genes, only a modest change in ELMO1 and PRKAG2 mRNA levels were observed in peripheral blood samples of CKD patients and controls, suggesting a poor correlation between DNA methylation changes and mRNA levels.

Study of epigenetic regulation occurring specifically in kidney cells is hampered by the heterogeneity of cell types genes showed changes in DNA methylation patterns be- 


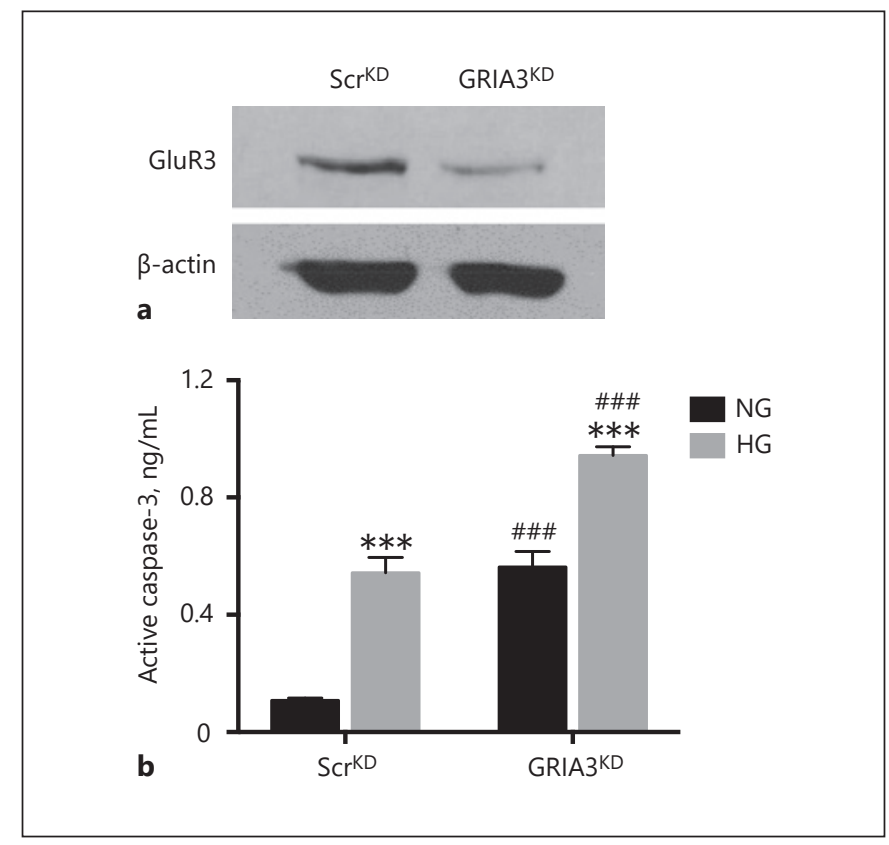

Fig. 6. Knockdown of GRIA3 exacerbates high glucose-induced podocyte apoptosis. a Representative western blot image of GluR3 in podocytes stably transduced with lentivirus expressing scrambled shRNA $\left(\mathrm{Scr}{ }^{\mathrm{KD}}\right)$ or GRIA3 shRNA (GRIA3 ${ }^{\mathrm{KD}}$ ). b Apoptosis was determined by measuring active caspase- 3 concentration in extracts from podocytes cultured under normal glucose (NG) or high glucose (HG) conditions. ${ }^{* * *} p<0.001$ vs. NG control; $\# \#$ \#\# 0.001 vs. respective glucose conditions of $\mathrm{Scr}{ }^{\mathrm{KD}}$.

present in kidney cortices or in isolated glomeruli. In order to overcome this limitation, a recent study analyzed the DNA methylation patterns in micro-dissected kidney tubular epithelial cells, which represented mainly the proximal tubules [30]. It showed significant cytosine methylation profiles for CKD kidneys and that differentially methylated loci are enriched in kidney-specific enhancer regions. To determine the podocyte-specific epigenetic changes in diabetic kidneys, we compared the DNA methylation profile in conjunction with mRNA sequencing in immortalized human podocytes cultured in either high or normal glucose conditions. Our analysis identified GRIA3 as one of two genes that were hypermethylated and associated with decreased mRNA expression in HG conditions.

We further examined the function of GRIA3, as several lines of evidence show that various glutamate receptors play a key role in podocyte biology [17]. Podocytes have been shown to contain neuron-like functional synaptic vesicles, which are glutamate-containing vesicles
[31], and in nonneuronal cells, glutamate acts as an extracellular signaling mediator. Glutamatergic signaling through NMDA receptor in podocytes has been reported to contribute to the function of the glomerular filtration barrier [21]. Interestingly, mice lacking the metabotropic glutamate receptor 1 (mGluR1) also developed albuminuria and glomerular damage [20], and selective mGluR1/5 agonist (S)-3,5-dihydroxyphenylglycine (DHPG) attenuated albuminuria and podocyte apoptosis in two proteinuric animal models [32]. The effect of DHPG is thought to be mediated by activation of cyclic AMP and CREB (cyclic AMP response element binding protein), resulting in restoration of mitochondrial function. In addition, nephrin was shown to interact with glutamine receptor in podocytes [33]. We now show that GRIA3 is epigenetically regulated in podocytes under HG conditions, and that GluR3, encoded by GRIA3, confers protection against podocyte apoptosis in HG conditions. Recent reports indicate that GluR3 promotes cell growth through activation of MAPK pathway [27]. Therefore, GluR3 may be a survival factor for podocytes through activation of the MAPK pathway [34, 35].

In summary, through combined DNA methylation and RNA sequencing profiling in human podocytes cultured under diabetic and normal conditions, we have identified novel genes, such as GRIA3, that may be involved in the pathogenesis of DKD.

\section{Acknowledgements}

J.C.H. is supported by NIH 1R01DK078897, NIH 1R01DK088541, and VA Merit Award; K.L. is supported by R01DK117913.

\section{Statement of Ethics}

The authors have no ethical conflicts to disclose.

\section{Disclosure Statement}

The authors declare that they have no competing financial interests.

\section{Author Contributions}

Z.L., H.C., and F.Z. performed experiments; W.Z. performed genomic data analysis, K.L. and J.C.H. designed the study and wrote the manuscript. 


\section{References}

1 Kato M, Natarajan R. Diabetic nephropathy - emerging epigenetic mechanisms. Nat Rev Nephrol. 2014 Sep;10(9):517-30.

2 Nathan DM; DCCT/EDIC Research Group. The diabetes control and complications trial/ epidemiology of diabetes interventions and complications study at 30 years: overview. Diabetes Care. 2014;37(1):9-16.

3 Chen Z, Miao F, Paterson AD, Lachin JM, Zhang L, Schones DE, et al.; DCCT/EDIC Research Group. Epigenomic profiling reveals an association between persistence of DNA methylation and metabolic memory in the DCCT/EDIC type 1 diabetes cohort. Proc Natl Acad Sci USA. 2016 May;113(21):E300211.

4 Meyer TW, Bennett PH, Nelson RG. Podocyte number predicts long-term urinary albumin excretion in Pima Indians with Type II diabetes and microalbuminuria. Diabetologia. 1999 Nov;42(11):1341-4.

5 Steffes MW, Schmidt D, McCrery R, Basgen JM; International Diabetic Nephropathy Study Group. Glomerular cell number in normal subjects and in type 1 diabetic patients. Kidney Int. 2001 Jun;59(6):2104-13.

6 Pagtalunan ME, Miller PL, Jumping-Eagle S, Nelson RG, Myers BD, Rennke HG, et al. Podocyte loss and progressive glomerular injury in type II diabetes. J Clin Invest. 1997 Jan; 99(2):342-8.

7 Macconi D, Bonomelli M, Benigni A, Plati T, Sangalli F, Longaretti L, et al. Pathophysiologic implications of reduced podocyte number in a rat model of progressive glomerular injury. Am J Pathol. 2006 Jan;168(1):42-54.

8 Tharaux PL, Huber TB. How many ways can a podocyte die? Semin Nephrol. 2012 Jul; 32(4):394-404

9 Basson MA, Akbulut S, Watson-Johnson J, Simon R, Carroll TJ, Shakya R, et al. Sproutyl is a critical regulator of GDNF/RET-mediated kidney induction. Dev Cell. 2005 Feb;8(2): 229-39.

10 Susztak K, Böttinger EP. Diabetic nephropathy: a frontier for personalized medicine. J Am Soc Nephrol. 2006 Feb;17(2):361-7.

11 Petermann AT, Pippin J, Krofft R, Blonski M, Griffin S, Durvasula R, et al. Viable podocytes detach in experimental diabetic nephropathy: potential mechanism underlying glomerulosclerosis. Nephron Exp Nephrol. 2004 98(4):e114-23.

12 Kang YS, Li Y, Dai C, Kiss LP, Wu C, Liu Y Inhibition of integrin-linked kinase blocks podocyte epithelial-mesenchymal transition and ameliorates proteinuria. Kidney Int. 2010 Aug;78(4):363-73.
13 Lipsky RH, Goldman D. Genomics and variation of ionotropic glutamate receptors. Ann N Y Acad Sci. 2003 Nov;1003(1):22-35.

14 Kumar J, Mayer ML. Functional insights from glutamate receptor ion channel structures. Annu Rev Physiol. 2013;75(1):313-37.

15 Wu Y, Arai AC, Rumbaugh G, Srivastava AK, Turner G, Hayashi T, et al. Mutations in ionotropic AMPA receptor 3 alter channel properties and are associated with moderate cognitive impairment in humans. Proc Natl Acad Sci USA. 2007 Nov;104(46):18163-8.

16 Yuan H, Low CM, Moody OA, Jenkins A, Traynelis SF. Ionotropic GABA and Glutamate Receptor Mutations and Human Neurologic Diseases. Mol Pharmacol. 2015 Jul; 88(1):203-17.

17 Dryer SE. Glutamate receptors in the kidney. Nephrol Dial Transplant. 2015 Oct;30(10): 1630-8.

18 Shen J, Wang R, He Z, Huang H, He X, Zhou $J$, et al. NMDA receptors participate in the progression of diabetic kidney disease by decreasing Cdc42-GTP activation in podocytes. J Pathol. 2016 Oct;240(2):149-60.

19 Kim EY, Anderson M, Dryer SE. Sustained activation of $\mathrm{N}$-methyl-D-aspartate receptors in podoctyes leads to oxidative stress, mobilization of transient receptor potential canonical 6 channels, nuclear factor of activated $\mathrm{T}$ cells activation, and apoptotic cell death. $\mathrm{Mol}$ Pharmacol. 2012 Oct;82(4):728-37.

20 Puliti A, Rossi PI, Caridi G, Corbelli A, Ikehata M, Armelloni S, et al. Albuminuria and glomerular damage in mice lacking the metabotropic glutamate receptor 1. Am J Pathol. 2011 Mar;178(3):1257-69.

21 Giardino L, Armelloni S, Corbelli A, Mattinzoli D, Zennaro C, Guerrot D, et al. Podocyte glutamatergic signaling contributes to the function of the glomerular filtration barrier. J Am Soc Nephrol. 2009 Sep;20(9):192940.

22 Saleem MA, O’Hare MJ, Reiser J, Coward RJ, Inward CD, Farren T, et al. A conditionally immortalized human podocyte cell line demonstrating nephrin and podocin expression. J Am Soc Nephrol. 2002 Mar;13(3):630-8.

23 Fu J, Wei C, Lee K, Zhang W, He W, Chuang $\mathrm{P}$, et al. Comparison of Glomerular and Podocyte mRNA Profiles in Streptozotocin-Induced Diabetes. J Am Soc Nephrol. 2016 Apr; 27(4):1006-14.

24 Ozawa H, Ashizawa S, Naito M, Yanagihara M, Ohnishi N, Maeda T, et al. Paired-like homeodomain protein ESXR1 possesses a cleavable C-terminal region that inhibits cyclin degradation. Oncogene. 2004 Aug;23(39): 6590-602.
25 Yanagihara M, Ishikawa S, Naito M, Nakajima J, Aburatani H, Hatakeyama M. Pairedlike homeoprotein ESXR1 acts as a sequencespecific transcriptional repressor of the human K-ras gene. Oncogene. 2005 Sep;24(38) 5878-87.

26 Nakajima J, Ishikawa S, Hamada J, Yanagihara M, Koike T, Hatakeyama M. Anti-tumor activity of ESX1 on cancer cells harboring oncogenic K-ras mutation. Biochem Biophys Res Commun. 2008 May;370(1):189-94.

27 Wei CH, Wu G, Cai Q, Gao XC, Tong F, Zhou $\mathrm{R}$, et al. MicroRNA-330-3p promotes cell invasion and metastasis in non-small cell lung cancer through GRIA3 by activating MAPK/ ERK signaling pathway. J Hematol Oncol. 2017 Jun;10(1): 125.

28 Ripka S, Riedel J, Neesse A, Griesmann H, Buchholz M, Ellenrieder V, et al. Glutamate receptor GRIA3 - target of CUX1 and mediator of tumor progression in pancreatic cancer. Neoplasia. 2010 Aug;12(8):659-67.

29 Smyth LJ, McKay GJ, Maxwell AP, McKnight AJ. DNA hypermethylation and DNA hypomethylation is present at different loci in chronic kidney disease. Epigenetics. 2014 Mar;9(3):366-76.

30 Ko YA, Mohtat D, Suzuki M, Park AS, Izquierdo MC, Han SY, et al. Cytosine methylation changes in enhancer regions of core pro-fibrotic genes characterize kidney fibrosis development. Genome Biol. 2013;14(10):R108.

31 Rastaldi MP, Armelloni S, Berra S, Calvaresi N, Corbelli A, Giardino LA, et al. Glomerular podocytes contain neuron-like functional synaptic vesicles. FASEB J. 2006 May;20(7): 976-8.

32 Gu L, Liang X, Wang L, Yan Y, Ni Z, Dai H, et al. Functional metabotropic glutamate receptors 1 and 5 are expressed in murine podocytes. Kidney Int. 2012 Mar;81(5):458-68.

33 Li M, Armelloni S, Ikehata M, Corbelli A, Pesaresi M, Calvaresi N, et al. Nephrin expression in adult rodent central nervous system and its interaction with glutamate receptors. J Pathol. 2011 Sep;225(1):118-28.

34 Hale LJ, Welsh GI, Perks CM, Hurcombe JA, Moore S, Hers I, et al. Insulin-like growth factor-II is produced by, signals to and is an important survival factor for the mature podocyte in man and mouse. J Pathol. 2013 May; 230(1):95-106.

35 Sieber J, Wieder N, Clark A, Reitberger M, Matan S, Schoenfelder J, Zhang J, Mandinova A, Bittker JA, Gutierrez J, Aygun O, Udeshi N, Carr S, Mundel P, Jehle AW, Greka A: GDC0879, a BRAF(V600E) Inhibitor, Protects Kidney Podocytes from Death. Cell Chem Biol 2018 Feb 15;25(2):175-184.e4. 